\title{
A DISTANCE ESTIMATE ON THE BOUNDARY OF A CONFORMAL DEFORMATION OF THE UNIT BALL SATISFYING A HARNACK INEQUALITY AND THE GEHRING-HAYMAN PROPERTY
}

\section{Timo Tossavainen}

University of Eastern Finland, School of Applied Educational Science and Teacher Education P.O. Box 86, FI-57101 Savonlinna, Finland; timo.tossavainen@uef.fi

\begin{abstract}
We establish some bounds for the natural distance function on the boundary of such a conformal deformation of the unit ball $\mathbf{B}^{n}, n \geq 2$, that satisfies a Harnack inequality and the condition of the Gehring-Hayman theorem. The construction is useful especially for those points for which the radial limit exists.
\end{abstract}

\section{Introduction}

A conformal deformation of the unit ball $\mathbf{B}^{n}, n \geq 2$, is a mapping of type $f:=\mathrm{Id}:\left(\mathbf{B}^{n}, g_{0}\right) \rightarrow\left(\mathbf{B}^{n}, d_{\rho}\right)$ where $g_{0}$ is the canonical metric of the Euclidean unit ball $\mathbf{B}^{n}$ and $d_{\rho}$ is the conformal metric derived from a continuous density $\rho: \mathbf{B}^{n} \rightarrow \mathbf{R}_{+}$ in the usual way:

$$
\operatorname{length}_{\rho}(\gamma)=\int_{\gamma} \rho(z)|d z|
$$

for a curve $\gamma$ in $\mathbf{B}^{n}$, and

$$
d_{\rho}(x, y)=\inf _{\gamma} \operatorname{length}_{\rho}(\gamma) \quad \text { for } x, y \in \mathbf{B}^{n},
$$

where the infimum is taken over all rectifiable curves joining $x$ and $y$ in $\mathbf{B}^{n}$. This metric extends also to the boundary of the unit ball via the usual limit process, see Section 6 in [2].

It is also possible to define a natural measure $\mu_{\rho}$ by setting

$$
\mu_{\rho}(E)=\operatorname{Vol}_{\rho}(E)=\int_{E} \rho^{n} d m_{n} \text { for a Borel set } E \subset \mathbf{B}^{n},
$$

where $m_{n}$ denotes the $n$-dimensional Lebesgue measure. Deformations of this kind are originally motivated by the theory of (quasi)conformal mappings. We refer the reader to [1], [2] and [3] for more information and concrete examples of conformal metrics.

In our setting we assume that the density $\rho$ satisfies a Harnack inequality, i.e., there exists a constant $A \geq 1$ so that

$$
\frac{1}{A} \leq \frac{\rho(x)}{\rho(y)} \leq A
$$

doi:10.5186/aasfm.2010.3509

2000 Mathematics Subject Classification: Primary 30C65. theorem.

Key words: Conformal deformation, conformal metric, Harnack inequality, Gehring-Hayman 
whenever $y \in B\left(x, \frac{1}{2}(1-|x|)\right)$ for some $x \in \mathbf{B}^{n}$. This is equivalent to assuming that the identity mapping $f$ above is uniformly quasi-symmetric in each Whitney ball $B\left(x, \frac{1}{2}(1-|x|)\right)$.

Following [3], we also define the isodiametric profile of $\left(\mathbf{B}^{n}, d_{\rho}\right)$ as a function $\eta_{\rho}:\left[0, \operatorname{diam}_{\rho}\left(\mathbf{B}^{n}\right)\right] \rightarrow[0, \infty]$,

$$
\eta_{\rho}(r)=\sup \left\{\mu_{\rho}(D): D \subset \mathbf{B}^{n} \text { and } \operatorname{diam}_{\rho}(D) \leq r\right\}
$$

In [2] it is shown that a large variety of conformal deformations satisfies, in addition to (1.1), also the condition $\eta_{\rho}(r) \leq C r^{n}$ for all $r>0$, which is equivalent to so-called volume growth condition, i.e., there exists a constant $B \geq 1$ so that

$$
\mu_{\rho}\left(B_{\rho}(x, r)\right) \leq B r^{n} \quad \text { for all } x \in \mathbf{B}^{n} \text { and } r>0 .
$$

Here $B_{\rho}(x, r)$ denotes an open ball with center $x$ and radius $r$ in the metric $d_{\rho}$. Further, in [2, Theorem 3.1] it is also shown that under assumptions (1.1) and (1.2) the following version of the Gehring-Hayman theorem is valid.

Theorem 1.1. (Gehring-Hayman Theorem) If $\gamma$ is hyperbolic geodesic in $\mathbf{B}^{n}$, then

$$
\int_{\gamma} \rho d s \leq C d_{\rho}(x, y)
$$

where $C=C(A, B, n)>0$ is a constant depending only on the constant $A, B$ and $n$ in (1.1) and (1.2).

For the moment, it is not precisely known how much one can relax the volume growth condition from (1.2) and still gain the Gehring-Hayman theorem. It is noticed in [6] that, for small $r$, the condition (1.2) is essentially optimal for the only known method to verify the Gehring-Hayman theorem in this kind of setting but, on the other hand, known examples of densities that satisfy (1.1) and not the Gehring-Hayman property, i.e., the condition of the Gehring-Hayman theorem, assume volume growth remarkably more extensive than (1.2).

Another point of view to the topic follows from that the conditions (1.1) and (1.2) - and hence the Gehring-Hayman property, too - admit that, at least, a set of Hausdorff dimension zero may blow up, cf. [2, Theorem 4.4]. (We say that the deformation mapping $f$ blows up at a point $z \in \partial \mathbf{B}^{n}$ if $\lim _{x \rightarrow z} d_{\rho}(0, x)=\infty$.) However, the examples of densities for which (1.1) holds but the Gehring-Hayman property does not seem to be related to points at which the deformation function blows up. For example, in the plane such an example is the $\rho$ for which $\rho(x)=1 /(1-|x|)$ along a fixed ray and elsewhere as small as (1.1) allows for some $A>2$.

In [1], [3] and [5] the size of the boundary of the unit ball under different conformal deformations is studied. Especially, it is shown in [5] that the boundary will not blow up in large parts if we assume (1.1) and loosen the condition (1.2) to some extent. For example, if $A>2$ and $\eta_{\rho}(r)=o\left(r^{n}(\log r)^{p}\right)$ as $r \rightarrow \infty$ for some $p>0$, then $f$ cannot blow up on a subset $E \subset \partial \mathbf{B}^{n}$ of positive $h$-measure of gauge

$$
h(t)=\frac{1}{\log \left(\frac{1}{t}\right)^{p+n-1}} .
$$

Further, if $A=2$, a much weaker growth condition on the isodiametric profile is sufficient for the previous conclusion: it is enough to assume that $\eta_{\rho}(r)=o\left(r^{n+p}\right)$ as $r \rightarrow \infty$. Unfortunately, also the relationship between the Gehring-Hayman property and the blowing-up-phenomenon is not yet completely clear. 
In this paper we shall establish bounds for geodesic lengths and distances on the boundary of the deformed unit ball using a simple cone construction. A similar construction has been applied, e.g., in [4]. The first result assumes only a Harnack inequality, the second one also the Gehring-Hayman property and hence it extends Lemma 1.7 in [7].

Theorem 1.2. Let $\rho: \mathbf{B}^{n} \rightarrow \mathbf{R}_{+}$be a continuous density satisfying (1.1). Then there exist constants $C^{\prime}=C^{\prime}(A) \in(0,1)$ and $C^{\prime \prime}=C^{\prime \prime}(A) \geq 1$ so that for every $x, y \in \partial \mathbf{B}^{n}$ we have

$$
C^{\prime} \operatorname{length}_{\rho}(\gamma) \leq \delta \leq C^{\prime \prime} \operatorname{length}_{\rho}(\gamma)
$$

where $\gamma$ is the hyperbolic geodesic joining $x$ and $y$ in $\mathbf{B}^{n}$,

$$
\delta=\max \left\{\operatorname{diam}_{\rho}([(1-h) x, x)), \operatorname{diam}_{\rho}([(1-h) y, y))\right\}
$$

in which $h=\frac{1}{2}|x-y|$ and $[(1-h) x, x)$ is a ray from $(1-h) x$ to $x$.

Theorem 1.3. Let $\delta$ and $\rho$ be such as in Theorem 1.2 and suppose also that $\int_{\gamma} \rho d s \leq C d_{\rho}(x, y)$ for some constant $C>0$. Then there exist constants $C^{\prime}=$ $C^{\prime}(A) \in(0,1)$ and $C^{\prime \prime}=C^{\prime \prime}(A, C) \geq 1$ so that for every $x, y \in \partial \mathbf{B}^{n}$ we have

$$
C^{\prime} d_{\rho}(x, y) \leq \delta \leq C^{\prime \prime} d_{\rho}(x, y) \text {. }
$$

Theorems 1.2 and 1.3. are useful especially for those points for which the radial limit exists. For example, for a quasiconformal mapping $f$, the integrability of

$$
\rho(x)=\left(\frac{1}{m_{n}\left(B_{x}\right)} \int_{B_{x}} J_{f} d m_{n}\right)^{1 / n}
$$

on a radius implies the existence of the radial limit and thus the existence of radial limits capacity-a.e. on the boundary, cf. [2, p. 649] and [8].

\section{The proofs of Theorem 1.2 and $\mathbf{1 . 3}$}

In [7, Lemma 1.3], it is given a proof for that the $\rho$-diameter of a Stolz cone is bounded by a constant depending only on $A$ of the Harnack inequality, the thickness of the cone, and the $\rho$-diameter of axis of the cone (The result was originally mentioned in [2]). More precisely, for $x \in \partial \mathbf{B}^{n}$ and the union

$$
\text { Cone }(x, \lambda, h)=\bigcup\{B(t x, \lambda(1-t)): 1-h \leq t<1\},
$$

where $\lambda \in(0,1)$ and $h \in(0,1]$, there exists a constant $C=C(A, \lambda)>0$ such that

$$
\operatorname{diam}_{\rho}(\operatorname{Cone}(x, \lambda, h)) \leq C \operatorname{diam}_{\rho}([(1-h) x, x)) \quad \text { for } h \in(0,1] .
$$

Choose now $\lambda=0.5$ and set the Stolz cones of height $h=|x-y| / 2$ at both $x$ and $y$. Then

$$
\operatorname{length}\left(\gamma \cap \operatorname{Cone}\left(x, \frac{1}{2}, h\right)\right)=\operatorname{length}\left(\gamma \cap \operatorname{Cone}\left(y, \frac{1}{2}, h\right)\right) \geq \frac{1}{4} \operatorname{length}(\gamma)
$$

and the part of $\gamma$ that is not in either of the cones can be covered with the chain of, at most, three carefully chosen Whitney balls that also join the cones to each others.

Suppose next that $\delta$ is given by the cone $F$ at, say, $x$. From (2.2) it follows that there are Whitney balls $B_{z}$ in $F$ for which

$$
\operatorname{length}\left(\gamma \cap B_{z}\right) \geq \frac{1}{2} \operatorname{diam}\left(B_{z}\right) .
$$


Let $F^{\prime}$ denote the largest union of the Whitney balls in $F$ for which (2.3) holds. Then clearly $t x \in F^{\prime}$ for $t \rightarrow 1$.

The Harnack inequality (1.1) implies that for every curve $\tilde{\gamma}$ in any Whitney ball $B_{z}$, we have

$$
\frac{1}{A} \rho(z) \operatorname{length}(\tilde{\gamma}) \leq \operatorname{length}_{\rho}(\tilde{\gamma}) \leq A \rho(z) \operatorname{length}(\tilde{\gamma})
$$

and, moreover, for $B_{z} \subset F^{\prime}$,

$$
\frac{1}{A} \rho(z) \operatorname{length}\left(\gamma \cap B_{z}\right) \leq \operatorname{diam}_{\rho}\left(B_{z}\right) \leq 2 A \rho(z) \operatorname{length}\left(\gamma \cap B_{z}\right) .
$$

Thus

$$
\frac{1}{A^{2}} \operatorname{length}_{\rho}\left(\gamma \cap B_{z}\right) \leq \operatorname{diam}_{\rho}\left(B_{z}\right) \leq 2 A^{2} \operatorname{length}_{\rho}\left(\gamma \cap B_{z}\right) .
$$

The existence of $C^{\prime}=C^{\prime}(A) \in(0,1)$ follows now from (2.1) and (2.2). Further, the triangle inequality implies now that there is a constant $C^{\prime \prime}=C^{\prime \prime}(A)$ such that

$$
\operatorname{diam}_{\rho}\left(F^{\prime}\right) \leq C^{\prime \prime} \operatorname{length}_{\rho}(\gamma)
$$

Moreover, by (2.2), it takes a chain of at most three Whitney balls to join every $x \in F \backslash F^{\prime}$ to $F^{\prime}$. Therefore there is another constant $C^{\prime \prime}=C^{\prime \prime}(A)$ such that

$$
\operatorname{diam}_{\rho}(F) \leq C^{\prime \prime} \operatorname{length}_{\rho}(\gamma) \text {. }
$$

From this, (2.1) and (2.4) it follows that there exists a constant $C^{\prime \prime}=C^{\prime \prime}(A)$ such that

$$
\delta \leq C^{\prime \prime} \operatorname{length}_{\rho}(\gamma)
$$

Theorem 1.2 is now proven. Theorem 1.3 follows directly from Theorem 1.2, the definition of $d_{\rho}$ and the Gehring-Hayman property.

\section{References}

[1] Bonk, M., and P. Koskela: Conformal metrics and size of the boundary. - Amer. J. Math. 124:6, 2002, 1247-1287.

[2] Bonk, M., P. Koskela, and S. Rohde: Conformal metrics on the unit ball in Euclidean space. - Proc. London Math. Soc. 77:3, 1998, 635-664.

[3] Hanson, B., P. Koskela, and M. Troyanov: Boundary behavior of the quasi-regular maps and the isodiametric profile. - Conform. Geom. Dyn. 5, 2001, 81-99.

[4] Koskela, P., and T. Tossavainen: Pathwise connectivity of a conformal boundary. - Bull. London Math. Soc. 35:5, 2003, 645-650.

[5] Nieminen, T., and T. Tossavainen: Boundary behaviour of conformal deformations. - Conform. Geom. Dyn. 11, 2007, 56-64.

[6] Nieminen, T., and T. Tossavainen: Conformal metrics on the unit ball: the Gehring-Hayman property and the volume growth. - Conform. Geom. Dyn. 13, 2009, 225-231.

[7] Tossavainen, T.: On the connectivity properties of the $\rho$-boundary of the unit ball. - Ann. Acad. Sci. Fenn. Math. Diss. 123, 2000.

[8] ZorIČ, V. A.: Angular boundary values of quasiconformal mappings of a ball. - Dokl. Akad. Nauk. SSSR 177, 1967, 771-773; English transl.: Soviet Math. Dokl. 8, 1967, 1492-1494. 